\title{
Hero: Automated Detection System for Prescription Stimulant Overdose via AI-Based Emotion Inference, Metabolite Detection, and Biometric Measurement
}

\author{
Divya Nori \\ Milton High School, Milton, GA, USA \\ Email: divyanori8@gmail.com
}

How to cite this paper: Nori, D. (2020) Hero: Automated Detection System for Prescription Stimulant Overdose via AI-Based Emotion Inference, Metabolite Detection, and Biometric Measurement. Open Journal of Applied Sciences, 10, 791-816. https://doi.org/10.4236/ojapps.2020.1012056

Received: November 10, 2020

Accepted: December 19, 2020

Published: December 22, 2020

Copyright $\odot 2020$ by author(s) and Scientific Research Publishing Inc. This work is licensed under the Creative Commons Attribution International License (CC BY 4.0).

http://creativecommons.org/licenses/by/4.0/

\begin{abstract}
Over the past year, approximately 10,000 Americans have died by psychostimulant overdose, and over $50 \%$ of these deaths were caused by prescription stimulant misuse. A comprehensive approach to detect a drug overdose in the environment where it occurs is imperative to reduce the number of prescription stimulant overdose-related deaths. Teenagers are at the highest risk for prescription stimulant overdose, so this study proposes a multi-factor overdose detection system named Hero which is designed to noninvasively operate within the context of a teen's life. Hero monitors five factors that indicate stimulant abuse: extreme mood swings, presence of amphetamine metabolite in sweat excreted from the fingertip, heart rate, blood pressure, and respiration rate. An algorithm to detect extreme mood swings in a teen's outgoing SMS messages was developed by collecting over 3.6 million tweets, creating groups of tweets for euphoria and melancholy using guidelines adapted from DSM-5 criteria, and training six Artificial Intelligence models. These models were used to create a dual-model-based extreme mood swing detection algorithm that was accurate $96 \%$ of the time. A biochemical strip, which consisted of a diagnostic measure that changes color when in contact with amphetamine metabolite and a control measure that changes color when the appropriate volume of sweat is excreted, was created. A gold nanoparticle-based diagnostic measure and $\mathrm{pH}$-based control measure were evaluated individually and on the overall strip. The diagnostic measure had an accuracy of 90.62\% while the control measure had $84.38 \%$ accuracy. Lastly, a vital sign measurement algorithm was built by applying photoplethysmography image processing techniques. A regression model with height, age, and gender features was created to convert heart rate to blood pressure, and the final algo-
\end{abstract}


rithm had an accuracy of $97.86 \%$. All five of these factors work together to create an accurate and easily integrable system to detect overdoses in real-time and prevent prescription stimulant abuse-related deaths.

\section{Keywords}

Addiction Science, Behavioral Science, Artificial Intelligence, Biochemistry

\section{Introduction}

Prescription stimulants are drugs intended to treat attention deficit hyperactivity disorder (ADHD) and narcolepsy by increasing dopamine and norepinephrine activity [1]. According to a survey conducted in 2020 by the National Institute on Drug Abuse, 6 percent of U.S. high school seniors reported nonmedical use of Adderall, the most common brand-name of prescription stimulants [2]. Although prescription stimulant misuse is a problem that can affect individuals of all demographics, adolescents and young adults are at the highest risk [3]. While prescription stimulants have been coined as "party" drugs and "weight loss" drugs, adolescents most commonly abuse it as a "cognitive steroid" [4]. Students are able to study for long hours, retain more information, and focus more attentively with the help of spiked norepinephrine levels [1]. If used when medically necessary at the correct dosage, prescription stimulants are inherently safe medications, but the line between intended use and abuse can often be blurred. These drugs are highly addictive and can lead to substance use disorder (SUD) and overdose [1]. According to the 2018 CDC Annual Surveillance Report of drug-related risks and outcomes, approximately 10,000 Americans died by psychostimulant overdose in past years and over $50 \%$ were caused by prescription stimulant misuse [5]. The relative ease with which these medications can be obtained on school campuses contributes to the increasing number of overdose cases among young people [6].

Looking at prescription drug overdose prevention more broadly, current research involves development of overdose-reversal therapies and drug antagonist-based therapeutics [7] [8]. However, administering an overdose-reversal therapy hinges on the teen being at a medical facility at the right time. One of the most common locations where drug misuse occurs, especially adolescent drug abuse, is in a bedroom alone [9]. Therefore, it may take hours before the situation is found by another individual, the overdose patient is taken to a medical facility, and appropriate intervention begins. The critical actions that must be taken after an overdose occurs, which includes administering CPR if necessary and calling first responders, may not occur until the situation has escalated beyond the scope of medical treatment. A real-time, non-invasive, and accurate framework to detect a drug overdose in the environment where it occurs is imperative to reducing the number of prescription stimulant overdose-related 
deaths in teens.

This study focuses on the development of a system to detect dangerous, overdose-nearing consumption of Adderall and similar prescription amphetamines. The system was designed for prescription amphetamines rather than illicit drugs or opioids because cocaine, heroin, opioids, and similar drugs are often taken for the purpose of achieving a "high". It is unlikely that a user of illicit drugs or opioids would willingly use an overdose detection system. However, the purpose of prescription amphetamines is to increase attentiveness rather than to reach dangerous consumption levels. It is more likely that an adolescent or young adult who consumes prescription amphetamines would use a real-time overdose detection system as a safety-net. Currently, few real-time detection systems exist, and these frameworks rely on single-factor analysis. The most promising results show that based on active sonar monitoring utilizing signals emitted by a smartphone, an individual's respiration rate can be determined, and abnormal respiration rate values indicate an overdose [10]. While this prior work is important in its demonstration of real-time monitoring, overdoses are extremely complex and analyzing just one physical manifestation compromises accuracy. A multi-factor approach taking emotional, biochemical, and physical signs of prescription amphetamine abuse into account is necessary to create an effective system. The factors chosen for examination in this study are extreme mood swings, presence of amphetamine metabolite in sweat excreted from the fingertip, and vital signs.

The following rationale supports the selection of these factors to detect a prescription amphetamine overdose. Prior literature shows that administration of high amphetamine dosages induces elation and euphoria followed by melancholy and anger [11] [12]. This extreme mood swing consists of the drug's colloquial "high," and the subsequent comedown which brings dysphoria [13]. While several drug classes have been shown to cause this emotional fluctuation, stimulant abusers are most subject to extreme mood swings [14]. This study proposes secure monitoring of a teen's outgoing text messages to detect an extreme mood swing and establish emotional indication of prescription stimulant abuse. Previous studies describe an algorithm that scores English phrases using one unsupervised machine learning model which is trained to detect polarity of emotional sentiment and calculate deviations [15]. However, prior algorithms were designed to detect routine mood fluctuations that teenagers commonly display. A framework that can distinguish between normal mood swings and an extreme mood swing has not been designed, partly because clinical guidelines have not been applied to capture emotional intensity in model training data. Additionally, the use of just one model in prior literature indicates a multiclass classifier with the ability to distinguish between positive, neutral, and negative sentiment. However, the creation of two separate binary classification models, one to distinguish between positive and neutral sentiment and another to distinguish between negative and neutral sentiment, was hypothesized to display higher accu- 
racy for the following reasons: relevant features can be selected separately for each classifier, the training groups will have balanced counts, and the optimal complexity can be selected for each classifier. This algorithm design consisting of two binary classification models is hereby referred to as a dual-model-based method. Hence, the first research question asks can a dual-model-based mood swing detection algorithm perform with higher accuracy than existing single-model-based algorithms? The independent variable was algorithm design and the dependent variable was accuracy.

The second factor chosen to detect a prescription amphetamine overdose was presence of amphetamine metabolite in sweat excreted from the fingertip. Fingertip sweat was selected for analysis rather than urine or blood because the overdose detection system being developed is intended to run noninvasively in real-time. Testing urine or blood would require a teen to deviate from daily routine while fingertip sweat analysis can be automated. Prior fingertip sweat-based drug screening protocols include lateral flow competition assays for presence of amphetamine [16]. However, by detecting amphetamine rather than amphetamine metabolite, false positives may result from touching the drug rather than testing for drug consumption. This study proposes detection of benzoic acid because in humans, $21 \%$ of the consumed amphetamine's mass is excreted as benzoic acid [17]. This amphetamine metabolite is detectable in sweat approximately two-hours after drug intake, and since peak drug effectiveness typically occurs three-hours after intake, detection of stimulant metabolite can be an effective preliminary indication to begin emotional and physical monitoring [18] [19]. One challenge that arises when detecting metabolite rather than the parent drug is that amphetamine metabolite is present at very low concentrations in sweat excreted from the fingertip [20]. An accurate metabolite detection method must be extremely sensitive, and therefore, this study investigated the use of gold nanoparticles (AuNPs) as a colorimetric probe. Large-sized AuNPs with strong color intensity improve biosensing capabilities, so the AuNPs served as the diagnostic measure on a biochemical test strip intended to analyze sweat excreted from the fingertip [21]. Additionally, a control measure was designed to ensure that an adequate volume of sweat was being analyzed. Based on prior literature, an average of $10 \mu \mathrm{l}$ of fingertip sweat is adequate for effective analysis, and this volume of fingertip sweat is excreted in approximately 1 minute [22] [23]. This study investigates the plausibility of a $\mathrm{pH}$-based test that displays a color change when a minimum of $10 \mu \mathrm{l}$ of sweat comes into contact with the biochemical strip, regardless of sweat composition. One research question investigated in this portion of the study was can an AuNP-based test display a significant difference in color when in contact with simulated metabolite-containing sweat versus simulated normal sweat? The second research question asks can the overall strip display a red-to-purple change when in contact with at least $10 \mu \mathrm{l}$ of metabolite-containing sweat? The independent variables were composition of sweat and volume of sweat, and the dependent variable was measurement of the color 
change.

Finally, increased heart rate, blood pressure, and respiration rate are common signs of prescription stimulant overdose [24]. One noninvasive method for continuous monitoring of these vital signs is photoplethysmography (PPG) imaging [25]. PPG works by emitting a light source onto a tissue, measuring the reflected light, and calculating the proportional blood volume variations [26]. Periodically reflecting light onto a user's fingertip using the phone's back camera and calculating vital signs whenever the user picks up their phone serves as an efficient real-time monitoring process for physical signs of overdose. However, existing PPG-based algorithms extrapolate blood pressure from heart rate using a standardized linear model [27]. This ignores variations due to gender, age, or height and may compromise vital sign estimation accuracy. Therefore, this study asks can a personalized PPG-based vital sign measurement algorithm perform with a higher accuracy than existing PPG-based algorithms? The independent variable was integration of personalized features and the dependent variable was accuracy.

The Hero system and paired mobile app incorporates all five of the factors as shown in Figure 1 for constant background monitoring of emotional, biochemical, and physical signs of overdose in a teen's day-to-day life. The purpose of this research is to implement novel detection methods into an efficient framework integrated that can be integrated into a teen or young adult's life.

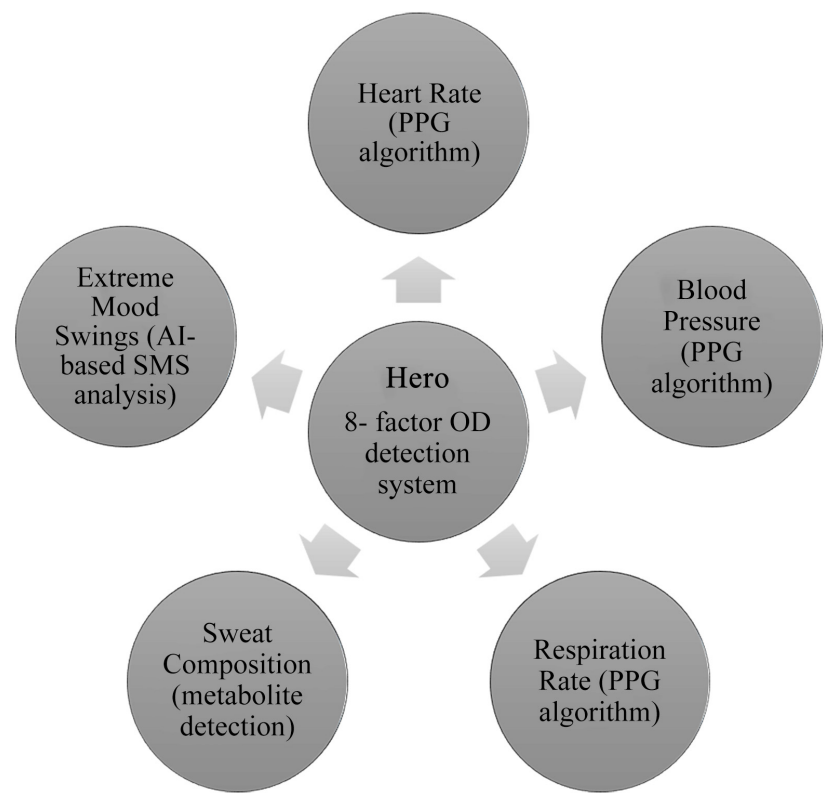

Figure 1. Hero integrates five factors to detect a drug overdose. The biochemical test strip is integrated onto a laptop mouse, and an Arduino color sensor monitors the strip for a color change. When a color change is detected for both the diagnostic and control measures, the Hero system begins monitoring the physical and emotional factors through the mobile app. This includes estimating vital signs using the personalized PPG-based algorithm and monitoring outgoing text messages for an extreme mood swing using the Artificial-Intelligence enabled dual-model-based algorithm. 


\section{Methods}

\subsection{Mood Swing Detection Algorithm}

A Python script using Tweepy, an open-source library used to access the Twitter API, was run for seven days. 3.6 million tweets and their corresponding twitter handles were gathered, and all tweets that were below a minimum of 100 characters, a retweet, or tweeted by a company/automated account were dropped. Each remaining tweet was then classified into one of the following groups: melancholic, euphoric, control, or irrelevant. The group classification criteria for melancholy were adapted from the DSM-5 Criteria for Major Depressive Disorder as shown in Table 1. Each guideline was adapted for single-tweet analysis, and if a tweet met three of the criteria, it was classified into the melancholy group.

Likewise, the classification guidelines for the euphoria group were adapted from the DSM-5 Criteria for a Bipolar I Disorder Manic Episode as shown in Table 2. If a tweet met three guidelines, it was classified as euphoric.

If a tweet met less than three criteria for either melancholy or euphoria, it was deemed irrelevant and dropped from the dataset. A tweet that met none of the criteria for melancholy or euphoria was classified into the control group. This clinical-based classification system was applied to ensure that the groups reflect

Table 1. The DSM-5 Criteria for Major Depressive Disorder was adapted to create guidelines appropriate for single-tweet analysis. If a tweet meets three of the six guidelines, it is classified into the melancholy group. This clinical-based classification system was applied to ensure that the groups reflect the intensity of emotion associated with a mood swing caused by high stimulant dosage.

\begin{tabular}{|c|c|}
\hline $\begin{array}{l}\text { DSM-5 Criteria for Major } \\
\text { Depressive Disorder }\end{array}$ & $\begin{array}{l}\text { Adapted Guidelines for Single-Tweet Analysis: } \\
\text { Melancholy Group }\end{array}$ \\
\hline $\begin{array}{l}\text { Depressed mood most of the day, nearly every } \\
\text { day. }\end{array}$ & $\begin{array}{l}\text { Tweet expresses sad, hopeless, or pessimistic } \\
\text { sentiment. }\end{array}$ \\
\hline $\begin{array}{l}\text { Markedly diminished interest or pleasure in all, } \\
\text { or almost all, activities most of the day, nearly } \\
\text { every day. }\end{array}$ & Tweet expresses lack of interest in activities. \\
\hline $\begin{array}{l}\text { Significant weight loss when not dieting or } \\
\text { weight gain, or decrease or increase in appetite } \\
\text { nearly every day. }\end{array}$ & Cannot interpret in a tweet. \\
\hline $\begin{array}{l}\text { A slowing down of thought and a reduction of } \\
\text { physical movement (observable by others, not } \\
\text { merely subjective feelings of restlessness or being } \\
\text { slowed down). }\end{array}$ & Cannot interpret in a tweet. \\
\hline Fatigue or loss of energy nearly every day. & Tweet expresses fatigue. \\
\hline $\begin{array}{l}\text { Feelings of worthlessness or excessive or } \\
\text { inappropriate guilt nearly every day. }\end{array}$ & Tweet expresses worthless/guilty sentiment. \\
\hline $\begin{array}{l}\text { Diminished ability to think or concentrate, or } \\
\text { indecisiveness, nearly every day. }\end{array}$ & $\begin{array}{l}\text { Tweet expresses diminished ability to } \\
\text { think/concentrate or indecisive sentiments }\end{array}$ \\
\hline $\begin{array}{l}\text { Recurrent thoughts of death, recurrent suicidal } \\
\text { ideation without a specific plan, or a suicide } \\
\text { attempt or a specific plan for committing } \\
\text { suicide. }\end{array}$ & $\begin{array}{l}\text { Tweet expresses suicidal intention or outlines a } \\
\text { specific plan for committing suicide. }\end{array}$ \\
\hline
\end{tabular}


Table 2. The DSM-5 Criteria for a Bipolar I Disorder Manic Episode was adapted to create guidelines appropriate for single-tweet analysis. If a tweet meets three of the six guidelines, it is classified into the euphoria group. This clinical-based classification system was applied to ensure that the groups reflect the intensity of emotion associated with a mood swing caused by high stimulant dosage.

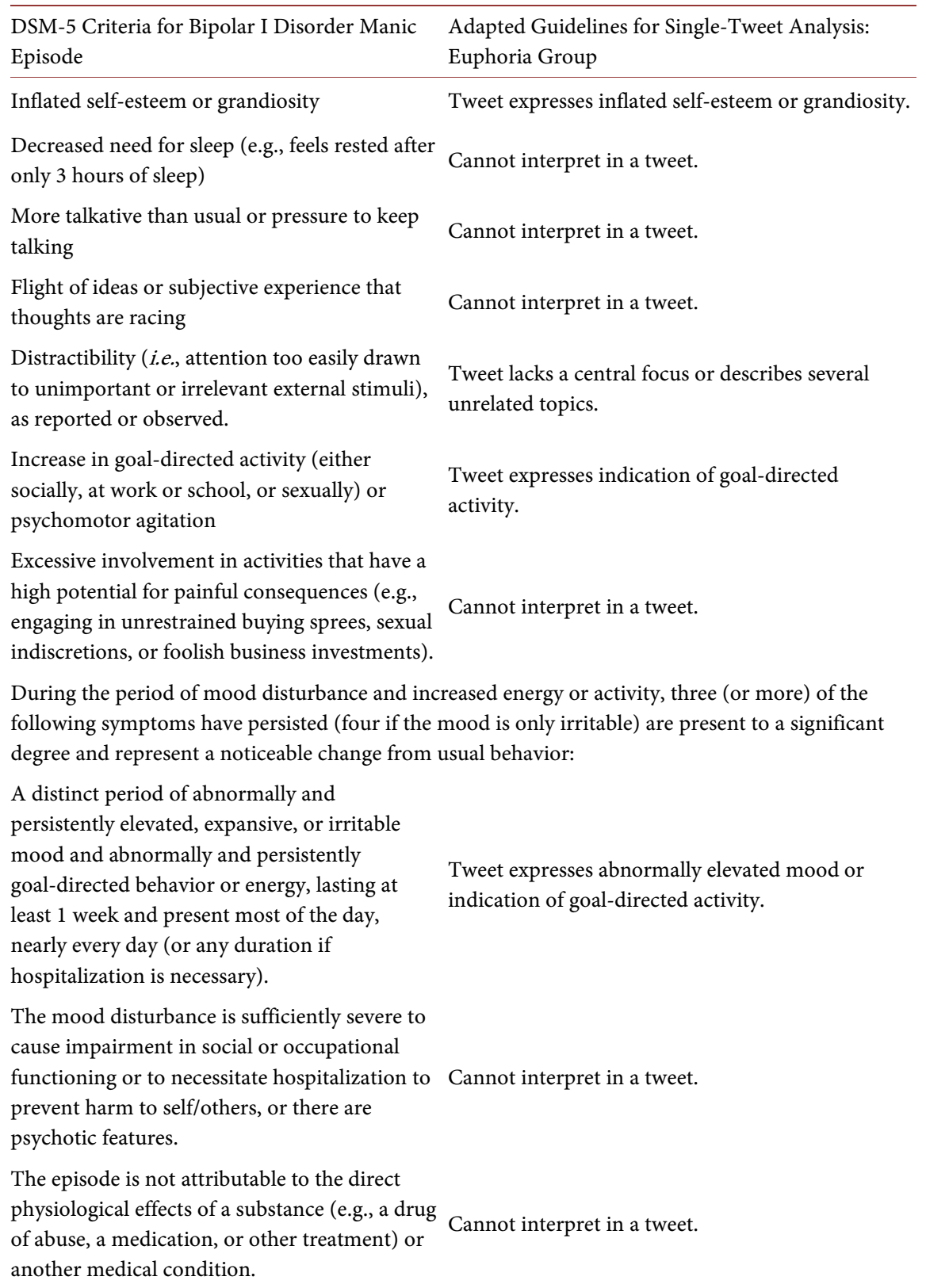

the intensity of emotion associated with a mood swing caused by high stimulant dosage. The final ground truth dataset, consisting of the two emotion groups and the control set, contained approximately 720,000 tweets. This data was read into the Python program for pre-processing which included replacing all Unicode characters and emoticons with an empty string to ensure that these special characters did not contribute to model training. Each group was then split into train $(80 \%)$ and test $(20 \%)$ groups, and the training group was split into 4 folds 
which supported cross-validation in model training. Each tweet in the training set was then split into unigrams (single words) and bigrams (sets of two words), and these $\mathrm{n}$-grams were stored in a vocabulary vector. Elements in the vocabulary vector that were present in at least $0.1 \%$ of tweets were considered features in model building. Once pre-processing was complete, two training matrices (one for melancholy and one for euphoria) with rows corresponding to tweets and columns corresponding to $\mathrm{n}$-gram features was created. A test matrix was also created following the same format. If the n-gram was present in the tweet, a 1 was placed in the cell. All other cells were set to 0 and stored in sparse matrix format. Finally, a dependent variable column was added containing the emotion classification of the tweet ( 0 for control; 1 for the matrix's respective emotion). The training matrices were used to build six different Artificial Intelligence models in Python, three melancholy identification models and three euphoria identification models. The first model type built was the Generalized Linear Model from the $\mathrm{H}_{2} \mathrm{O}$ library which is a hyperplane-based approach. The least absolute shrinkage and selection operator (LASSO) was used to create a parsimonious model and prevent overfitting. The next model type was the Gradient Boosting Machine which is a tree-based approach. Lastly, a Multilayer Perceptron which is a deep learning-based approach was built. After the models were created, the test matrix was used to calculate model metrics such as AUC and accuracy, sensitivity, specificity, and F1-Score for different thresholds. These model metrics were analyzed to identify a score threshold and design a dual-model-based mood swing detection algorithm. The algorithm monitors a teen's outgoing SMS messages, scores each one with both the euphoria and melancholy models, and uses these scores to detect an extreme mood swing. This dual-model-based algorithm was tested by simulating mood swings using messages from the euphoria and melancholy groups, and the number of true positives, true negatives, false positives, and false negatives were recorded.

\subsection{Biochemical Test Strip}

Biochemical strip design began by creating a $0.0010 \mathrm{M}$ stock solution of gold (III) ions from 1.0 gram of Hydrogen Tetrachloroaurate (III) Trihydrate. $20 \mathrm{~mL}$ of this stock solution was heated on a stirring hotplate until bubbles formed. After creating a $1 \%$ Trisodium Citrate Dihydrate solution separately, $2 \mathrm{~mL}$ of the citrate solution was added to the $20 \mathrm{~mL}$ stock solution on the hotplate. When the solution turned deep red (approximately ten minutes), it was removed from the hotplate and stored in a brown bottle because Hydrogen Tetrachloroaurate (III) Trihydrate is hygroscopic and reacts in visible light. Citrate synthesis was applied to create AuNPs because this synthesis procedure creates larger particles, and AuNPs with larger diameters are more sensitive [28]. Simulated metabolite-containing sweat was created by adding $0.5 \mathrm{~g} \mathrm{NaCl}$ (mimics natural salts in sweat) and $5 \mu \mathrm{l}$ of benzoic acid (metabolite) to $10 \mathrm{~mL}$ of distilled water. The same procedure was followed to create normal sweat, but benzoic acid was not added. The AuNP diagnostic measure was then evaluated in isolation using a spectro- 
photometer to record absorbance for the original AuNP solution, AuNP solution with metabolite-containing sweat (16 trials), and AuNP solution containing normal sweat (16 trials). The spectrophotometer was set to the wavelength at which the original AuNP solution absorbed most strongly $(500 \mathrm{~nm})$. Measuring at this $\lambda \max$ value helped ensure that the absorbance readings would be sensitive to changes as the volume of metabolite-containing sweat was incremented.

Next, the $\mathrm{pH}$-based control measure was evaluated in isolation. Using a strong acid $(\mathrm{HCl})$ and strong base $(\mathrm{NaOH})$, solutions at integer $\mathrm{pH}$ values from 0 to 8 were created using the serial dilution method. A pH probe was calibrated using buffers and utilized to finetune the $\mathrm{pH}$ of the created solutions. A few drops of anthocyanin indicator were transferred to each solution, resulting in a range of colored liquids. Using a spectrophotometer, two $\lambda$ max values were selected (one where acidic solution with indicator absorbed strongly and one where basic solution with indicator absorbed strongly), and absorbance at these two wavelengths was recorded for each colored solution. This data was used to determine the indicator's $\mathrm{pKa}$. This spectrophotometric procedure for a precise determination of $\mathrm{pKa}$ was used because the control measure must change color when in contact with just $10 \mu \mathrm{l}$ of sweat. Since the strip must be sensitive to such small volumes, an exact determination of solution volumes that must be present on the original strip was necessary. The overall strip was then evaluated (32 trials), and the trials are broken down in Figure 2.

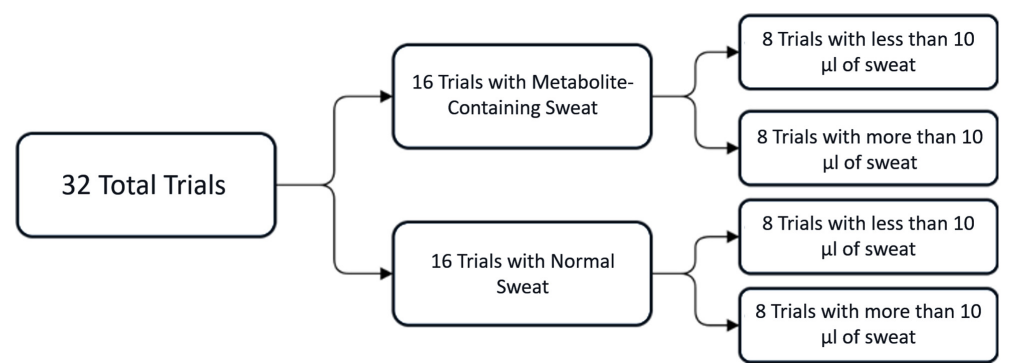

Figure 2. The 32 total trials consisted of 16 trials with metabolite-containing sweat and 16 trials with normal sweat. These groups were further divided based on volume of sweat (less than or more than $10 \mu \mathrm{l}$ ). The expected number of positives for both the diagnostic and control measure is 16 , and the expected number of negatives for both measures is 16 .

\subsection{Vital Sign Algorithm}

The vital sign algorithm was developed in Android Studio using the Java programming language. First, a PPG-based algorithm was built, and the algorithm began by turning on the phone's back camera with flash and taking a 5-second recording of the user's illuminated fingertip. This recording was then parsed and processed to obtain the RGB intensities of each frame. This is because during the cardiac cycle, when the heart beats, a wave of blood reaches the capillaries at the end of the fingertip. When the capillary is full, the RGB values of the frame will differ from when the capillary is empty. Red and green intensities were then stored in an array, and the Fast Fourier Transform (Math package in Java) was applied to obtain a resultant array. After filtering out noise from the resultant 
array, a peak detection algorithm was used to find all of the cardiac peaks in the signal. The R-R interval (time difference between peaks) was computed, and heart rate was then calculated by dividing this value by 60 . Publicly available data from the National Heart, Lung, and Blood Institute was then used to develop a regression model in Python with the following features: heart rate, height, age, and gender. The output of the regression model was a blood pressure measurement. Finally, the bandpass filter (from the Math package) was applied to the heart rate signal in order to reduce irregular sampling from the smart phone. The Fast Fourier Transform was re-applied to the modulated heart rate arrays and a clear peak was observed at each breath which allowed for calculation of respiration rate.

The vital sign algorithm was evaluated through analysis of regression model metrics and manual comparison to an accurate measurement method. 21 trials were conducted in which the author modulated my respiration from 7 to 35 breaths per minute. A program was written in Java to blink at a specific interval, keeping respiration rate constant. The number of actual breaths per minute was recorded, and for a 5-second interval during that minute, the photoplethysmography algorithm was run. The results from the actual counting and the algorithm were compared and statistical measures were computed.

\section{Results}

\subsection{Mood Swing Detection Algorithm}

Figure 3 displays the distribution of scores returned by the Generalized Linear Model (GLM) and Gradient Boosting Machine (GBM) for euphoria when analyzing new messages (test matrix). For GLM, the mean score of the messages displaying euphoria was 0.57 and the standard deviation was 0.15 , while the mean score of the control messages was 0.40 and the standard deviation was 0.16 . For GBM, the mean score of the messages displaying euphoria was 0.60 and the standard deviation was 0.16 , while the mean score of the control messages was 0.38 and the standard deviation was 0.16 . The GBM resulted in a much more bimodal distribution, indicating that this tree-based approach performed better than the hyperplane-based approach for euphoria.

Similarly, Figure 4 displays the distribution of scores returned by the Generalized Linear Model (GLM) for melancholy and Gradient Boosting Machine (GBM) for melancholy when analyzing messages designated for testing. For GLM, the mean score of the melancholic messages was 0.55 and the standard deviation was 0.15 , while the mean score of the control messages was 0.40 and the standard deviation was 0.13 . For GBM, the mean score of the melancholic messages was 0.60 and the standard deviation was 0.18 , while the mean score of the control messages was 0.36 and the standard deviation was 0.16 . Again, the GBM resulted in a much more bimodal distribution, indicating that this tree-based approach performed better than the hyperplane-based approach for melancholy. 


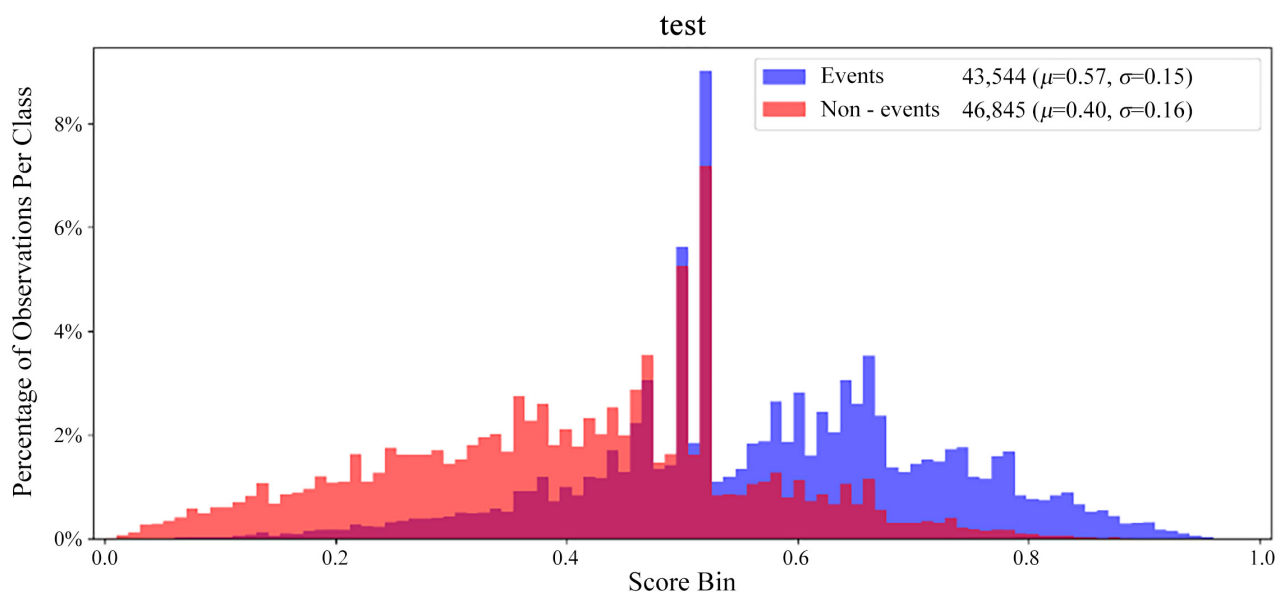

(a)

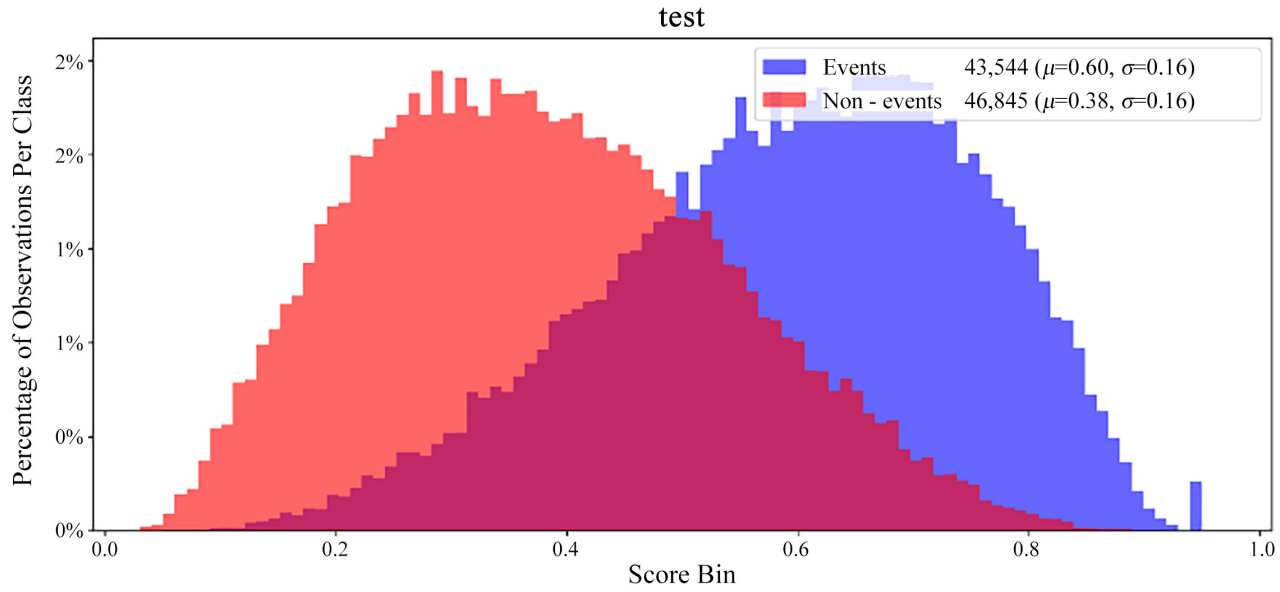

(b)

Figure 3. (a) The histogram shows the number of messages in each score bin by percentage of observations per class as scored by the GLM euphoria model. The number of messages in the euphoria test group was 43,544 and the number of messages in the control test group was 46,845 (randomly selected from a larger set to match number of euphoric messages). (b) The GBM resulted in a much more bimodal distribution in comparison to the GLM, indicating that this tree-based approach performed better than the hyperplane-based approach for euphoria.

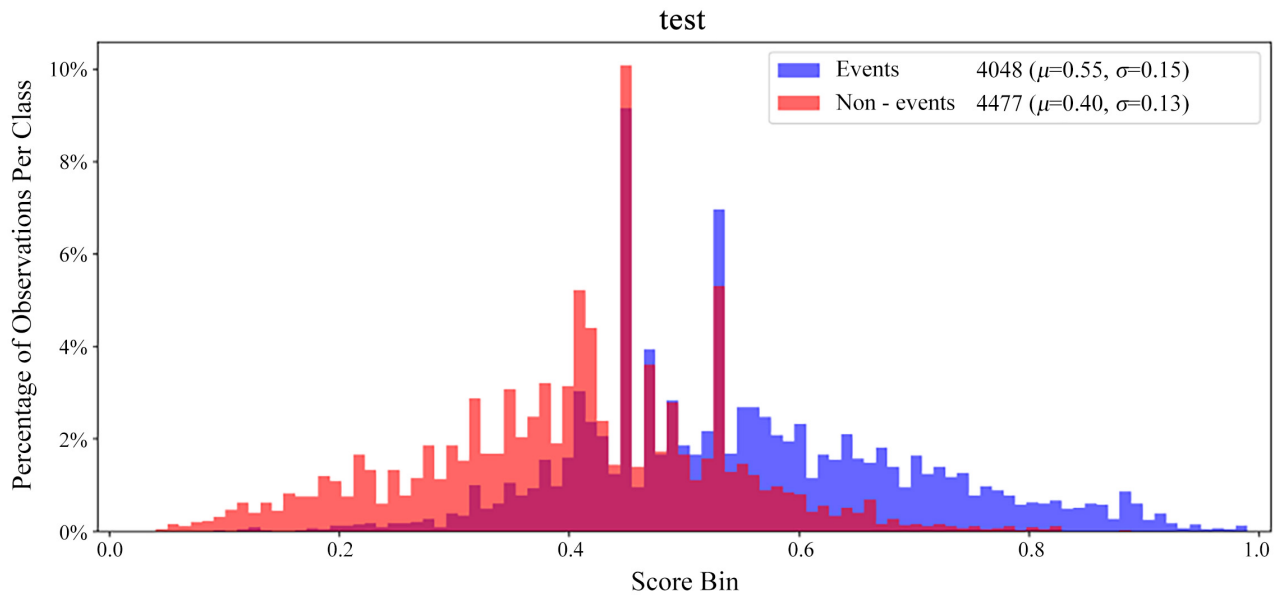

(a) 


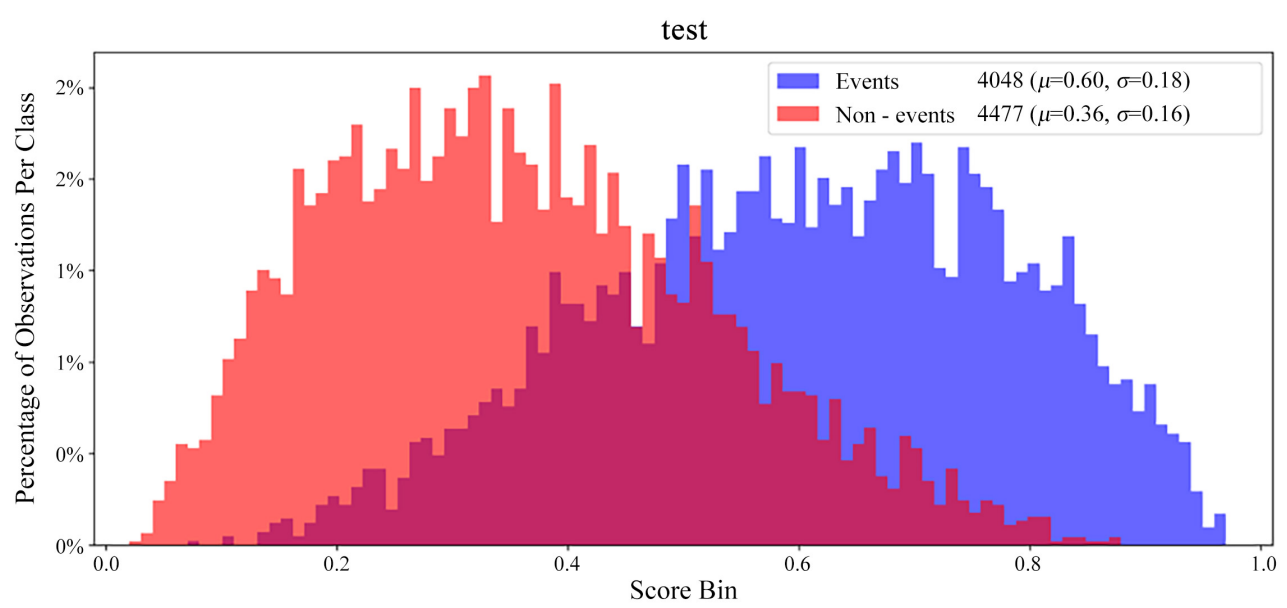

(b)

Figure 4. (a) The histogram shows the number of messages in each score bin by percentage of observations per class as scored by the GLM melancholy model. The number of messages in the melancholy test group was 4048 and the number of messages in the control test group was 4477 (randomly selected from a larger set to match number of melancholic messages). (b) The GBM for melancholy resulted in a much more bimodal distribution in comparison to the GLM, indicating that this tree-based approach performed better than the hyperplane-based approach for euphoria.

Area Under the Curve (AUC) was compared between the Generalized Linear Model (GLM), Gradient Boosting Machine (GBM), and Multilayer Perceptron (MLP) for both emotions. The Receiver Operating Characteristic (ROC) curve plots the False Positive Rate against the True Positive Rate. As shown in Figure 5, for euphoria, GLM testing had an AUC of 0.7852, GBM testing had an AUC of 0.8294, and MLP testing had an AUC of 0.8409 (5.57 percentage point increase from GLM to MLP). For melancholy, GLM testing had an AUC of 0.7762 , GBM testing had an AUC of 0.8384, and MLP testing had an AUC of 0.8618 (8.56 percentage point increase from GLM to MLP). The precision recall (PR) curves show that for euphoria, the average precision increased by 6.59 percentage points between GLM and MLP, and for melancholy, the increase was 8.10 percentage points. For both emotions, the multilayer perceptron displayed the highest AUC and average precision.
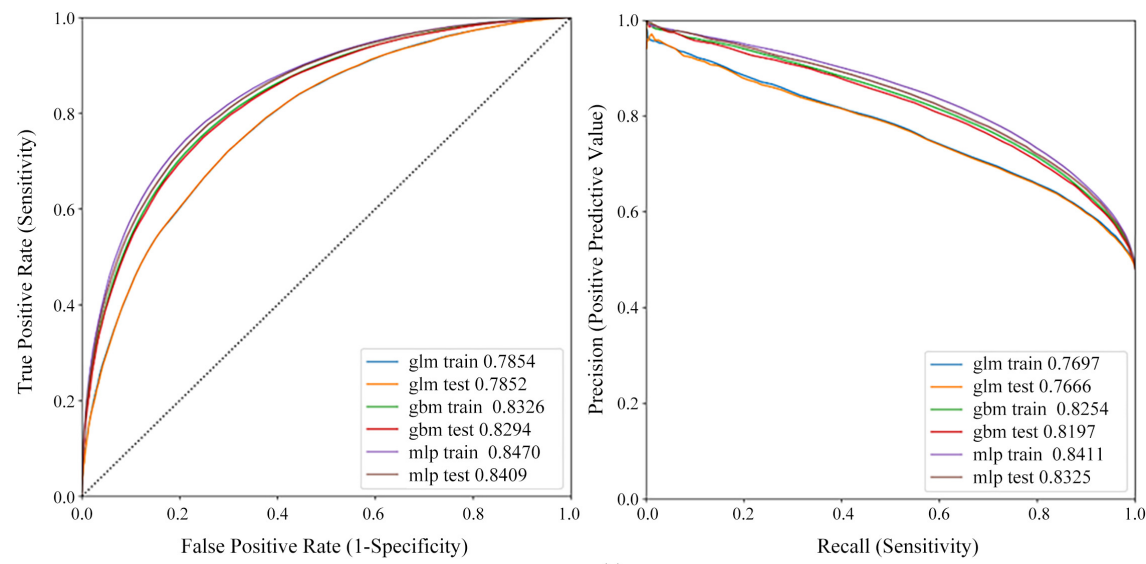

(a) 

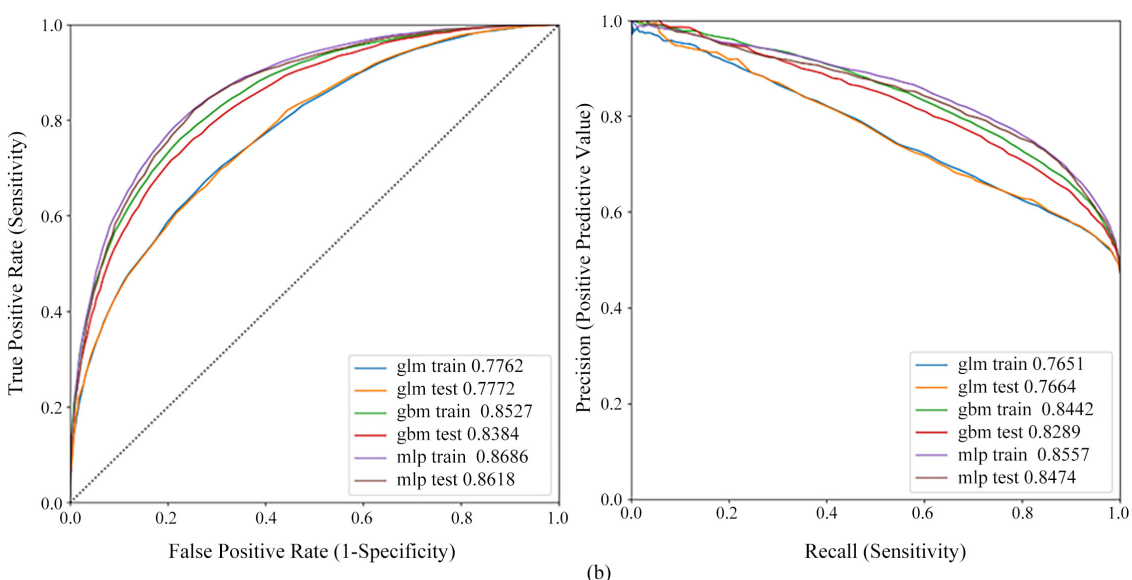

Figure 5. (a) The ROC curve plots the False Positive Rate (1 - specificity) against the True Positive Rate (sensitivity). The euphoria model that performed best was the multilayer perceptron with a test AUC of 0.8409. The precision-recall curve plots recall (sensitivity) against precision (positive predictive value). Of the euphoria models, the average precision was the highest for the multilayer perceptron. (b) The melancholy model with the highest AUC was the multilayer perceptron with a test AUC of 0.8618 . Of the melancholy models, the average precision was the highest for the multilayer perceptron.

To determine the optimal score threshold, the accuracy, sensitivity, and specificity of the GBM models across a range of possible thresholds was analyzed as shown in Figure 6. The score threshold is the value that differentiates between a message displaying the model's respective emotion and a message that lacks emotional intensity. For euphoria, as shown in 6, 0.51 was the score threshold with maximum accuracy, and 0.49 was the score threshold where sensitivity, specificity, and accuracy intersected (optimal). For melancholy, 0.49 was the score threshold with maximum accuracy, and 0.48 was the score threshold where all three measures are optimal.

The F1-Score is defined as the harmonic mean of a model's sensitivity and positive predictive value. For both GBM models, the F1-Score is maximized at a score threshold of 0.5. Therefore, the final score threshold was selected as 0.5 for both euphoria and melancholy. If the euphoria model returns a score higher than 0.5 for a message, euphoric sentiment has been identified. Similarly, if the melancholy model returns a score higher than 0.5 , melancholic sentiment has been identified.

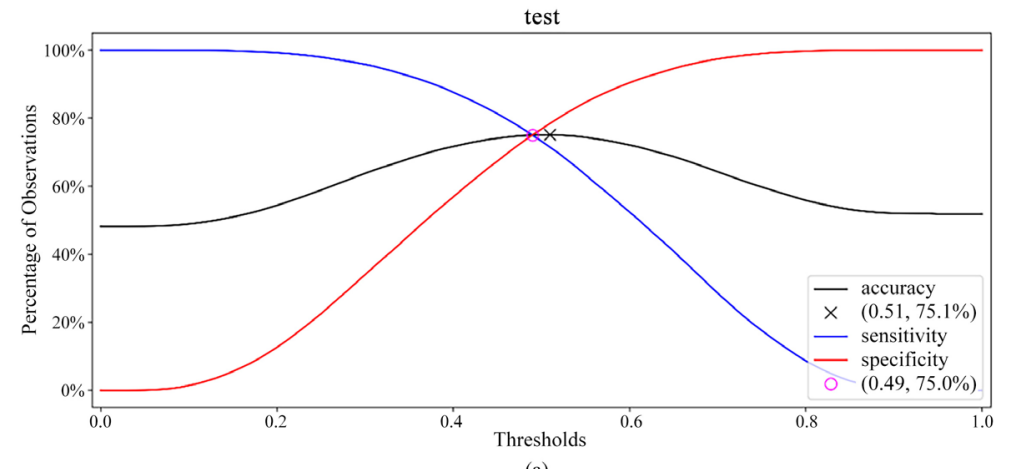

(a) 


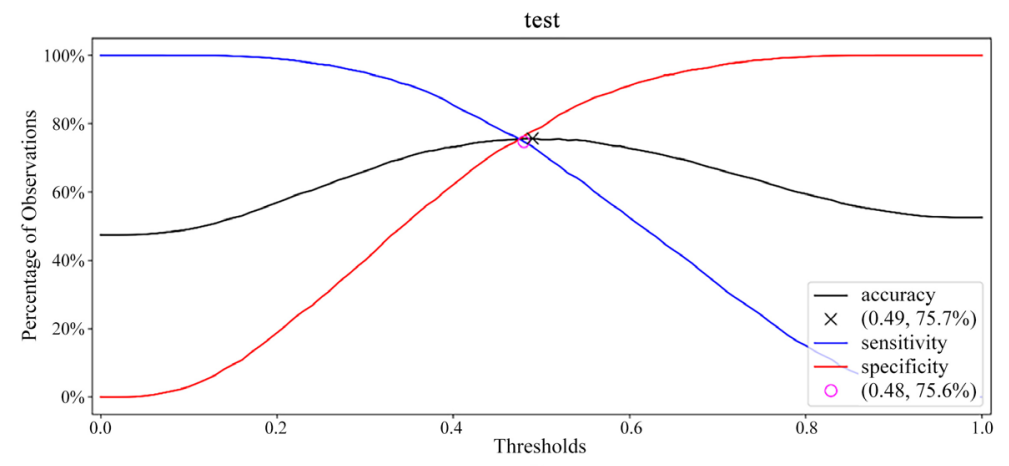

(b)

Figure 6. (a) As shown by the black "X" mark, 0.51 is the score threshold at which accuracy is maximized for the GBM euphoria model. The pink circle shows that a score threshold of 0.49 , the sensitivity, specificity, and accuracy intersect. (b) As shown by the black " $\mathrm{X}$ " mark, 0.49 is the score threshold at which accuracy is maximized for the GBM melancholy model. The pink circle shows that a score threshold of 0.48 , the sensitivity, specificity, and accuracy intersect.

Figure 7 shows the architecture of the multilayer perceptron (MLP). The structure of the MLP is similar to a biological neuron with model characteristics like node inputs, weight multiplication, and offset bias resembling dendrites, cell body processing, and synapse signal modulation. Since the MLP is architecturally similar to the human brain, it may be able to model these psychological processes more effectively and thus displays higher accuracies in comparison to GLM and GBM [29].

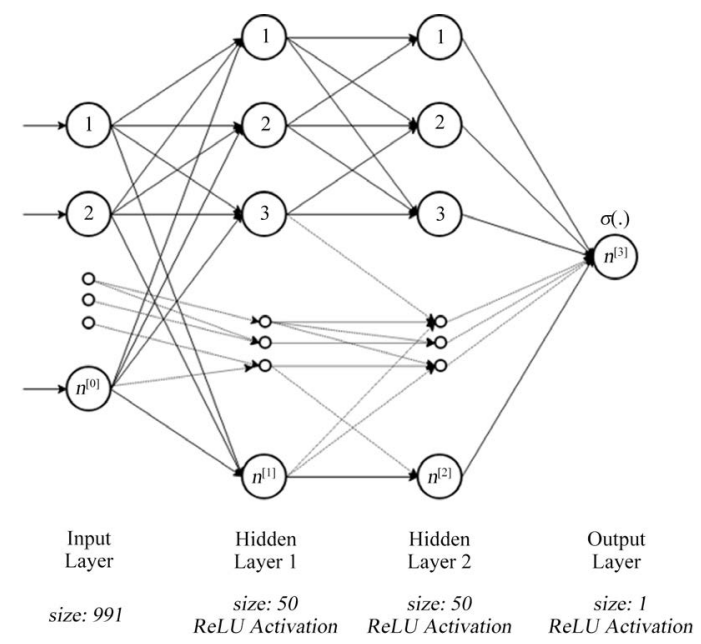

Figure 7. The MLP contains an input layer, two hidden layers, and an output layer. The Rectified Linear Unit (ReLU) activation function is used for the hidden layers, and the Sigmoid activation function is used for the output layer.

The final mood swing algorithm was evaluated with simulated extreme mood swings using messages from the emotion groups. Table 3 shows the number of true positives, false negatives, true negatives, and false positives. The dual-model-based algorithm's sensitivity was $92.0 \%$, specificity was $100.0 \%$, and accuracy was $96.0 \%$. 
Table 3. The mood swing detection algorithm returned 23 TP's, 2 FP's, 25 TN's, and 0 FN's. The sensitivity was $92.0 \%$, specificity was $100.0 \%$, and accuracy was $96.0 \%$.

\begin{tabular}{cc}
\hline Result & Counts \\
\hline True Positive (TP) & 23 \\
False Negative (FP) & 2 \\
True Negative (TN) & 25 \\
False Positive (FN) & 0 \\
Total Number of Simulated Mood Swings & 50 \\
\hline
\end{tabular}

\subsection{Biochemical Test Strip}

Table 4 displays the means and standard deviations of absorbance measurements taken at $500 \mathrm{~nm}$ for the original AuNP solution as well as solutions containing incremented amounts of metabolite-containing sweat combined with AuNP. The mean absorbance of the original AuNP solution was 0.24 at $500 \mathrm{~nm}$, and as expected, absorbance decreased as metabolite-containing sweat was added. The ratio of AuNP solution to sweat required for the most significant color change was determined by running Independent Samples T-tests to compare the original AuNP solution's absorbance to the experimental solutions' absorbance. As shown in Figure 8, a visible color change occurred almost instantaneously and persisted for at least 20 minutes.

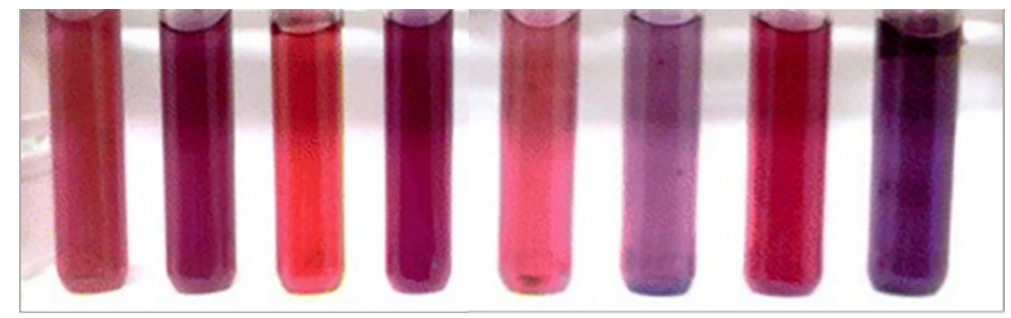

Figure 8. The 1st, 3rd, 5th, and 7th cuvettes show the original $2 \mathrm{~mL}$ of AuNP solution, and these solutions are all fairly red in color. The 2 nd cuvette displays the AuNP solution combined with $25 \mu$ l of metabolite-containing sweat, and the solution has a slightly purple tint. The 4 th, 6 th, and 8 th cuvettes display 2 $\mathrm{mL}$ AuNP solution combined with $50 \mu \mathrm{l}, 75 \mu \mathrm{l}$, and $100 \mu \mathrm{l}$ of simulated metabolite-containing sweat respectively, and as more simulated sweat is added, the purple tint intensifies. The last cuvette (8th) is a deep purple color.

Table 4. The mean absorbance across 4 trials at $500 \mathrm{~nm}$ of the original AuNP solution was 0.24 . When $25 \mu \mathrm{l}$ of simulated metabolite-containing sweat was added, the mean absorbance was 0.21 . For $50 \mu \mathrm{l}, 75 \mu \mathrm{l}$, and $100 \mu \mathrm{l}$, the absorbance values were $0.19,0.11$, 0.08 respectively. The largest absorbance difference was observed with $100 \mu$ of sweat.

\begin{tabular}{|c|c|c|c|c|c|}
\hline $\begin{array}{l}\text { Evaluation of AuNP Test } \\
\text { with simulated } \\
\text { metabolite-containing sweat }\end{array}$ & $\begin{array}{l}\text { Original AuNP } \\
\text { Solution }\end{array}$ & $\begin{array}{c}2 \mathrm{~mL} \text { AuNP } \\
\text { Solution }+25 \mu \mathrm{l} \\
\text { sweat }\end{array}$ & $\begin{array}{c}2 \mathrm{~mL} \text { AuNP } \\
\text { Solution }+50 \mu \mathrm{l} \\
\text { sweat }\end{array}$ & $\begin{array}{c}2 \mathrm{~mL} \text { AuNP } \\
\text { Solution }+75 \mu \mathrm{l} \\
\text { sweat }\end{array}$ & $\begin{array}{c}2 \mathrm{~mL} \text { AuNP } \\
\text { Solution }+100 \mu \mathrm{l} \\
\text { sweat }\end{array}$ \\
\hline Mean Absorbance (at $500 \mathrm{~nm}$ ) & 0.24 & 0.21 & 0.19 & 0.11 & 0.08 \\
\hline Standard Deviation of Absorbance & 0.01 & 0.04 & 0.03 & 0.03 & 0.02 \\
\hline t-test Value & NA & 1.46 & 3.16 & 8.22 & 14.3 \\
\hline
\end{tabular}


Similarly, absorbance at $500 \mathrm{~nm}$ was recorded for solutions combining AuNP solution and incremented values of normal sweat to ensure that a significant color change did not occur. The means, standard deviations, and T-test values are shown in Table 5. The original AuNP solution had a mean absorbance of 0.24 , and when $100 \mu \mathrm{l}$ of simulated normal sweat was added, the mean absorbance changed by only 0.01 .

Table 5. The mean absorbance across 4 trials at $500 \mathrm{~nm}$ of the original AuNP solution was 0.24 . When $25 \mu \mathrm{l}$ of simulated normal sweat was added, the mean absorbance was 0.24 . For $50 \mu \mathrm{l}, 75 \mu \mathrm{l}$, and $100 \mu \mathrm{l}$, the absorbance value was 0.23 for all three volumes.

\begin{tabular}{|c|c|c|c|c|c|}
\hline $\begin{array}{l}\text { Color Change Evaluation } \\
\text { of AuNP Test with normal } \\
\text { sweat at } 500 \mathrm{~nm}\end{array}$ & $\begin{array}{c}\text { Original AuNP } \\
\text { Solution (4 trials) }\end{array}$ & $\begin{array}{l}2 \mathrm{~mL} \text { AuNP Solution }+ \\
25 \mu \mathrm{l} \text { simulated normal } \\
\text { sweat (4 trials) }\end{array}$ & $\begin{array}{l}2 \mathrm{~mL} \text { AuNP Solution }+ \\
50 \mu \mathrm{l} \text { simulated normal } \\
\text { sweat ( } 4 \text { trials) }\end{array}$ & $\begin{array}{l}2 \mathrm{~mL} \text { AuNP Solution }+ \\
75 \mu \mathrm{l} \text { simulated normal } \\
\text { sweat ( } 4 \text { trials) }\end{array}$ & $\begin{array}{l}2 \mathrm{~mL} \text { AuNP Solution }+ \\
100 \mu \mathrm{l} \text { simulated normal } \\
\text { sweat ( } 4 \text { trials) }\end{array}$ \\
\hline $\begin{array}{l}\text { Mean Absorbance } \\
\quad \text { (at } 500 \mathrm{~nm} \text { ) }\end{array}$ & 0.24 & 0.24 & 0.23 & 0.23 & 0.23 \\
\hline $\begin{array}{c}\text { Standard Deviation of } \\
\text { Absorbance }\end{array}$ & 0.01 & 0.02 & 0.02 & 0.01 & 0.01 \\
\hline t-test Value & NA & 0 & 0.894 & 1.41 & 1.41 \\
\hline
\end{tabular}

To precisely determine anthocyanin $\mathrm{pH}$ indicator's $\mathrm{pKa}$ and optimize the control measure, one wavelength where an acidic solution mixed with indicator absorbed strongly and another wavelength where basic solution mixed with indicator absorbed strongly were identified. As shown in Figure 9, the two $\lambda \max$ values were $520 \mathrm{~nm}$ and $680 \mathrm{~nm}$.

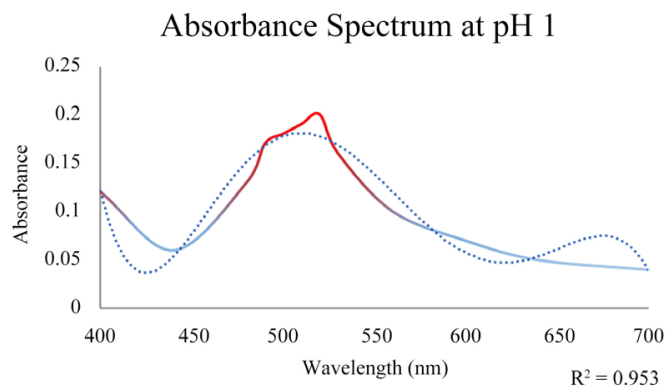

(a)

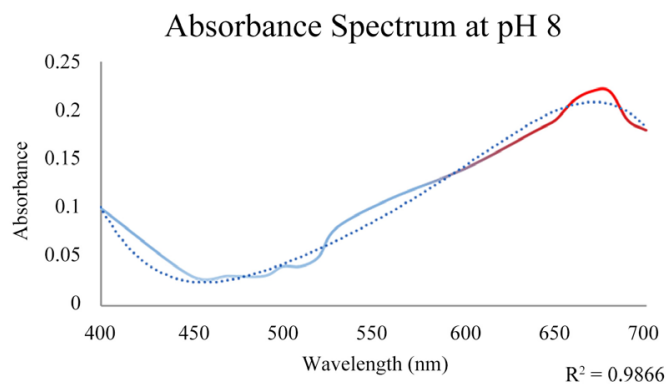

(b)

Figure 9. (a) The absorbance spectrum at $\mathrm{pH} 1$ shows a peak at $\lambda=520 \mathrm{~nm}$, indicating that acidic solutions mixed with anthocyanin indicator absorb most strongly at this wavelength. (b) The absorbance spectrum at $\mathrm{pH} 8$ shows a peak at $\lambda=680$ $\mathrm{nm}$, indicating that basic solutions mixed with anthocyanin indicator absorb most strongly at this wavelength. $\mathrm{pH} 8$ was selected to test basic solutions as supposed to a higher $\mathrm{pH}$ value because sweat typically cannot go beyond a $\mathrm{pH}$ of 8 . 
These two wavelengths were then used to record absorbance for solutions with indicator across a range of $\mathrm{pH}$ values, and background signal was subtracted. As shown in Figure 10, using these adjusted absorbance readings, the anthocyanin indicator's $\mathrm{pKa}$ was calculated as 4.35 . Based on the fact that anthocyanin indicator will change color at a $\mathrm{pH}$ of approximately $4.35,2.88 \mu \mathrm{l}$ of $0.0002 \mathrm{M} \mathrm{HCl}$ must originally be present on the strip so that when $10 \mu \mathrm{l}$ of sweat is excreted, the $\mathrm{pH}$ will surpass 4.35 and a red-to-purple change will occur. The $2.88 \mu \mathrm{H} \mathrm{HCl}$ volume was rounded down to $2.75 \mu \mathrm{l}$ to account for slightly acidic sweat, so for the control measure to display a distinct red to purple color change, $2.75 \mu \mathrm{l}$ of $0.0002 \mathrm{M} \mathrm{HCl}$ and a few drops of anthocyanin indicator must be on the strip originally.

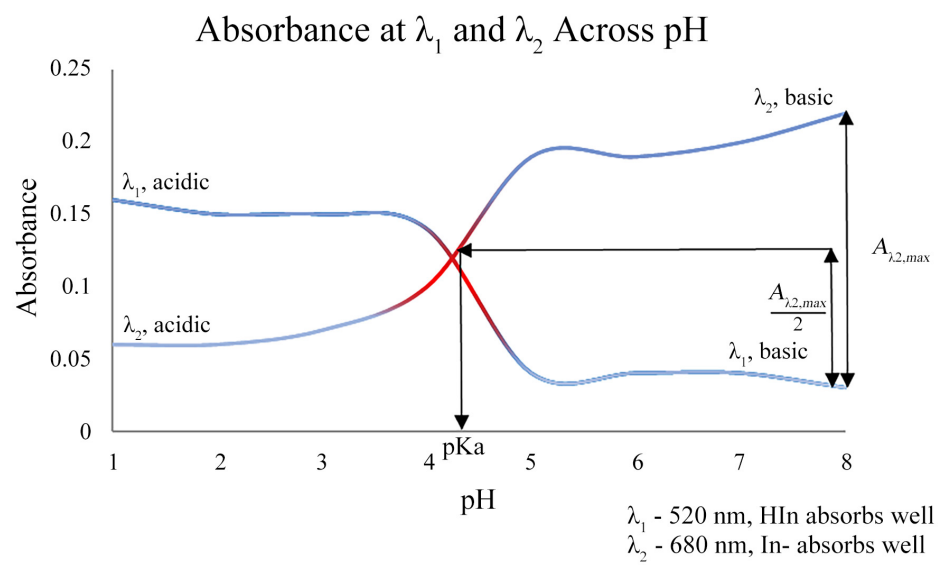

Figure 10. At $520 \mathrm{~nm}$ and $680 \mathrm{~nm}$, absorbance readings for solutions across a range of $\mathrm{pH}$ values were recorded. After plotting these values, the $\mathrm{pKa}$ was calculated to be $\sim 4.35$. The arrows drawn on the graph show how this pKa value was computed.

Table 6 shows the true positive (TP), false positive (FP), true negative (TN), and false negative (FN) counts when the overall strip was evaluated. For the diagnostic measure, the sensitivity was $93.75 \%$, specificity was $87.50 \%$, and accuracy was $90.62 \%$. For the control measure, the sensitivity was $87.50 \%$, specificity was $86.67 \%$, and accuracy was $84.38 \%$. Example strips after contact with metabolite-containing sweat and normal sweat are shown in Figure 11.

Table 6. The diagnostic measure displayed $15 \mathrm{TP}, 1 \mathrm{FN}, 14 \mathrm{TN}$, and 2 FP results. Therefore, the accuracy of the diagnostic measure was $90.62 \%$ across 32 trials. The control measure displayed $14 \mathrm{TP}, 2 \mathrm{FN}, 13 \mathrm{TN}$, and $3 \mathrm{FP}$ results. The accuracy of the control measure was $84.38 \%$ across 32 trials.

\begin{tabular}{ccc}
\hline Result & Counts (Diagnostic Measure) & Counts (Control Measure) \\
\hline True Positive (TP) & 15 & 14 \\
False Negative (FN) & 1 & 2 \\
True Negative (TN) & 14 & 13 \\
False Positive (FP) & 2 & 3 \\
Total Number of Samples & 32 & 32 \\
\hline
\end{tabular}




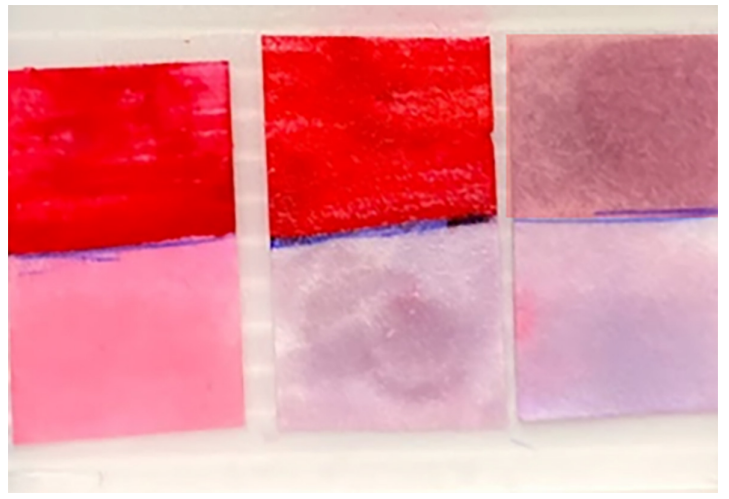

Figure 11. The bottom panel on the strip is the pH-based control measure (changes color no matter the sweat composition) and the top panel is the AuNP-based diagnostic measure. The left-most strip is the original strip, the middle strip shows the color after contact with normal sweat, and the right-most strip shows the color after contact with metabolite-containing sweat. As expected, the control measure changed color regardless of sweat composition and the diagnostic measure changed color in the presence of amphetamine metabolite.

In the Hero system, a color sensor monitors the biochemical strip for a color change. The strip is integrated onto a computer mouse. The sensor monitors the strip's area on the mouse, and if a color change is detected, the remaining components of the Hero system are activated. Two color sensor prototypes were built and tested as shown in Figure 12. Both prototypes contained an Arduino Uno board, bread board, photometric sensor, TFT display, WiFi module, and jumper wires.
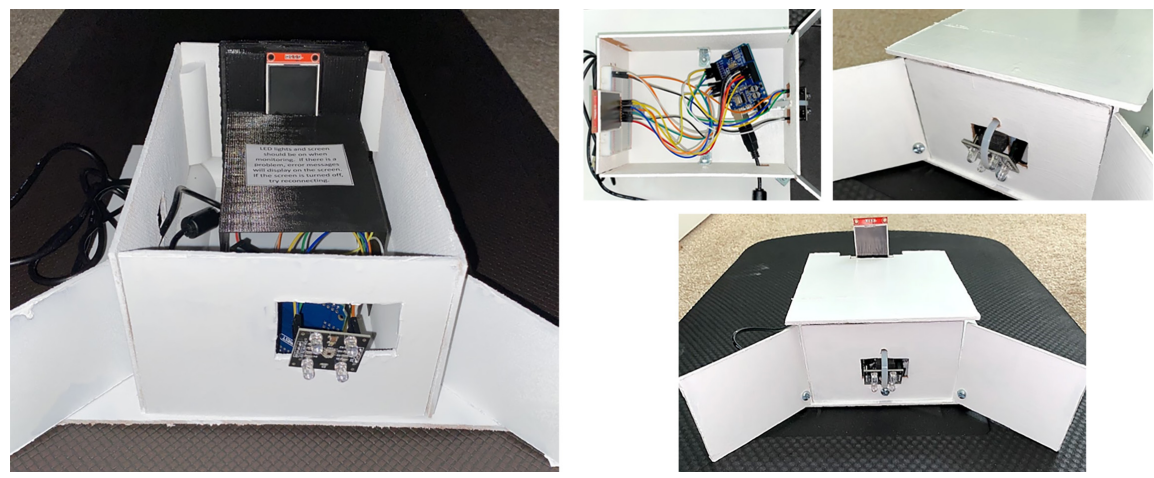

Figure 12. The first prototype (left) included a 3D-printed casing to host the TFT display and an opening through which the color can observe the mouse. The second prototype (right) included a method of controlling the angle at which the color sensor is positioned. This improves the sensor's sensing ability.

As shown in Table 7, the initial prototype was tested for percent error of sensing (average 6.2\%) and time taken to begin monitoring (average 4.55 seconds). A second prototype was created to increase the angle at which the sensor was positioned, and this helped increase sensing ability. The time improved by $41.2 \%$ (average 2.675 seconds) and percent error decreased by $62.5 \%$ (average $2.325 \%$ ). Testing was conducted using original chemical test strips 
(red), and recording the actual and measured RGB values. It was then changed for a strip in contact with metabolite-containing sweat and then a strip in contact with normal sweat. RGB values were measured again and average percent error was computed.

Table 7. Prototype 1 had an average percent error of $6.2 \%$ while Prototype 2 had an average percent error of $2.3 \%$, so the modification of sensor position improved sensing accuracy by 3.9 percentage points (62.5 percent decrease). Prototype 1 had an average response time of 4.55 seconds while Prototype 2 had an average response time of 2.675 seconds. This is a 1.88 second difference (41.2 percent decrease).

\begin{tabular}{ccc}
\hline Prototype & RGB Value Average Percent Error & Time Taken to Begin Monitoring (seconds) \\
\hline 1 & $6.4 \%$ & 4.5 \\
1 & $6.5 \%$ & 4.8 \\
1 & $6.1 \%$ & 4.4 \\
1 & $5.8 \%$ & 4.5 \\
2 & $2.2 \%$ & 2.9 \\
2 & $2.4 \%$ & 2.5 \\
2 & $2.3 \%$ & 2.6 \\
2 & $2.4 \%$ & 2.7 \\
\hline
\end{tabular}

\subsection{Vital Sign Algorithm}

Table 8 shows the results from evaluating the respiration rate component of the vital sign algorithm. The average algorithm reading in breaths per minute is shown. The overall mean absolute percentage error was $2.14 \%$, so the accuracy was $97.86 \%$. This statistical measure was chosen as supposed to root means squared error because each observation is weighted equally.

Table 8. The respiration rate component of the vital sign algorithm was evaluated by comparing algorithm readings to accurate respiration rate measurements. The mean absolute percent error was $2.14 \%$, so the accuracy was $97.86 \%$.

\begin{tabular}{ccc}
\hline Actual Rate (Breaths per Minute) & Average Algorithm Reading & Percent Error \\
\hline $7-11(9)$ & 9.25 & $+2.78 \%$ \\
$11-15(13)$ & 13.50 & $+3.85 \%$ \\
$15-19(17)$ & 16.75 & $-1.47 \%$ \\
$19-23(21)$ & 21.50 & $+2.38 \%$ \\
$23-27(25)$ & 25.75 & $+3.00 \%$ \\
$27-31(29)$ & 29.00 & $0.00 \%$ \\
$31-35(33)$ & 33.50 & $+1.52 \%$ \\
\hline
\end{tabular}

The vital sign regression model's coefficients are shown in Table 9. The heart rate feature had the largest coefficient of 4.065 , followed by age, height, and gender. All of the coefficients are positive, indicating that heart rate, height, age, 
and gender all have an effect on blood pressure. The model had an adjusted $\mathrm{R}$-squared value of 0.987 , and this measure is adjusted for the number of predictors in the model.

Table 9. The coefficients returned by the regression model are all positive, meaning they all have an effect on blood pressure. The largest coefficient of 4.065 corresponds to heart rate.

\begin{tabular}{cl}
\hline Coefficient & Value \\
\hline Heart Rate & 4.065 \\
Height & 0.321 \\
Age & 0.532 \\
Gender & 0.112 \\
\hline
\end{tabular}

\section{Discussion}

Based on the means and standard deviations of the scores returned by the Generalized Linear Model (GLM) for euphoria, the Independent Samples t-test value is 164.87 . This value is greater than the critical value at a $0.1 \%$ significance level, indicating that the GLM euphoria model displays a significant difference in scores between euphoric messages and control messages. Similarly, for the euphoria Gradient Boosting Machine (GBM), the t-test value is 206.56. This value is greater than the critical value, so the euphoria GBM displays a significant difference in scores and can identify euphoric sentiment. Because the GBM's t-test value is greater and the score output from GBM is more bimodally distributed, GBM performed better than GLM for euphoria. For the GLM melancholy model, the $\mathrm{t}$-test value is 49.01 , exceeding the critical value at a significance level of $0.1 \%$. The scores returned by the GBM melancholy model result in a t-test value of 64.79. The GBM's t-test value is greater than that of GLM, indicating that the GBM model performed better. Since the $t$-test value of both euphoria models are greater than the $\mathrm{t}$-test value of the melancholy models, the euphoria models are likely able to differentiate euphoric sentiment from a normal message more clearly than the melancholy models. However, all GLM and GBM t-test values exceed the critical values, indicating that when integrated into the Hero system, the models can detect extreme emotional sentiment in a teens' outgoing text messages. This is important for the dual-model-based algorithm because both models must be able to detect and quantify emotional polarity. Final mood swing detection algorithm testing resulted in an accuracy of $96.0 \%$ which exceeds the accuracies cited in prior literature by 4.3 percentage points [30].

The t-test value computed through comparison of the original AuNP solution and the AuNP solution combined with $25 \mu \mathrm{l}$ of simulated metabolite-containing sweat is 1.46, and at a significance level of 5\% and with 3 degrees of freedom, the color change is not significant. Similarly, the t-test values computed through comparison of the original AuNP solution and the AuNP solution combined with $50 \mu \mathrm{l}$ of simulated metabolite-containing sweat is 3.16 does not exceed the 
critical value. However, for the trials using $75 \mu \mathrm{l}$ and $100 \mu \mathrm{l}$ of metabolite-containing sweat, the t-test values are higher than the critical value, indicating that the diagnostic measure's color change is significant at these AuNP to sweat ratios. The change was most significant at a AuNP to sweat ratio of 20 to 1. Therefore, assuming that $10 \mu \mathrm{l}$ of sweat is excreted from the fingertip, $200 \mu \mathrm{l}$ of AuNP solution should be placed on the original strip for optimal results. All of the t-test values computed for trials using normal sweat are below the critical value, indicating that a significant color change did not occur when metabolite was not present. Through these results, the diagnostic measure's validity is displayed, illustrating that citrate-synthesized AuNPs are an effective colorimetric probe for amphetamine metabolite detection at microliter ranges. After determining the volumes of solutions that need to be placed on the original strip for optimal results $(200 \mu \mathrm{l}$ of AuNP solution for diagnostic measure and $2.75 \mu \mathrm{l}$ of $0.0002 \mathrm{M} \mathrm{HCl}+$ a few drops of anthocyanin indicator for control measure), these conclusions were applied to evaluate the overall strip. The control measure's accuracy of $84.38 \%$ provides preliminary confirmation that a $\mathrm{pH}$-based test can help avoid false positives and validate that an appropriate volume of sweat is being analyzed. The diagnostic measure's accuracy of $90.62 \%$ is also promising and shows that this method can be used more widely in on-site drug testing.

The final color sensor prototype had a $97.7 \%$ accuracy. One way that this value could be improved is by creating a field of detection as illustrated in Figure 13.

The vital sign algorithm had a $97.86 \%$ accuracy which is similar to other PPG-based algorithms designed to analyze a fingertip recording taken noninvasively. However, while this study is unable to confirm that the integration of personalized features improves PPG-based vital sign calculation, one hypothesis is that personalization would have a more beneficial impact for individuals who are below 18-years-old, above 65-years-old, below the 25 th percentile in height, or above the 75 th percentile in height. For these individuals who are not close to the median age/height, standardized heart rate to blood pressure conversions may be less accurate. Because the Hero system is targeted towards teens and young adults whose ages are below the median, a personalized vital sign algorithm would likely be more accurate in comparison to existing algorithms. Additionally, the coefficients returned by the blood pressure regression model are all positive, indicating that height, age, and gender all have an impact on blood pressure.

The Hero system integrates the dual-model-based mood swing detection algorithm, biochemical strip, and the personalized PPG-based vital sign measurement algorithm in the following way. The biochemical strip is placed on the button portions of a computer mouse, and the color sensor apparatus monitors this area for a color change from red to purple. While this prototype system is built with the strip on a computer mouse, the strip and color sensor could be placed in any area where the teen's fingertip makes repeated contact. When the sensor detects a color change, physical and emotional monitoring begins. The 
Hero app runs the vital sign measurement algorithm when the teen picks up their mobile device and saves heart rate, blood pressure, and respiration rate measurements. If these values are significantly higher than the teen's baseline values which are saved in a health questionnaire upon app download, there is indication of stimulant abuse. The total overdose score which is saved in Google's Firebase real-time database is updated appropriately. The app securely monitors the SMS messages that a teen's sends, and if an extreme mood swing is detected, the overdose score is updated. When the overdose score exceeds a specific threshold (set by the parent), an alert will be sent. The Hero prototype system is fully-functional, and sample app screens are shown in Figure 14.

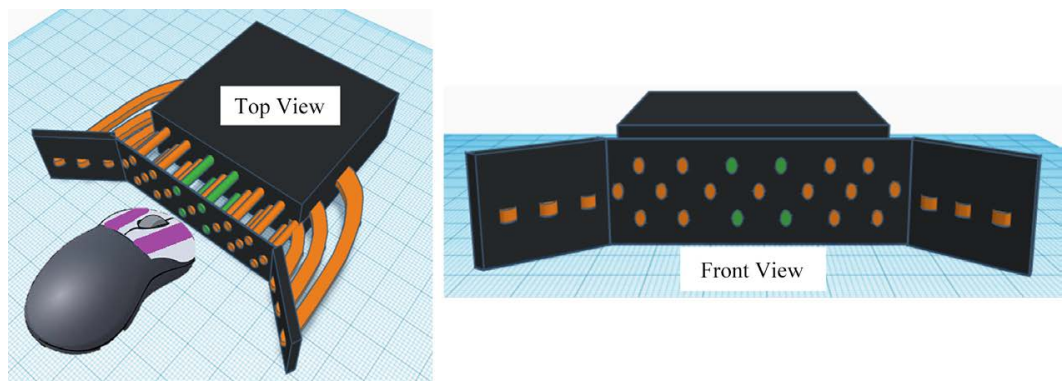

Figure 13. This schematic diagram, created using CAD software shows how sensors could be arranged in a hardware apparatus to monitor the computer mouse. The sensors shown in green are implemented in the existing prototype.
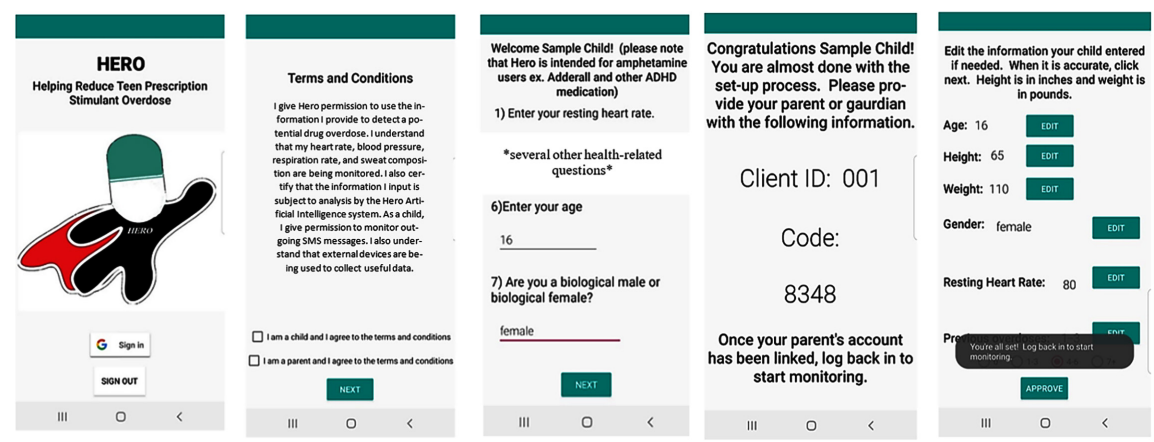

Figure 14. Screen 1 (left-most) shows a splash screen containing the Hero logo and Google sign-in functionality. When a parent or teen logs in, they are taken to Screen 2. Screen 2 (second from left) states Hero's terms and conditions and allows users to agree. If the user is a teen, they are taken to the health information questionnaire shown on Screen 3 (middle). On this screen, they enter their resting heart rate, height, age, resting respiration rate, and gender. Once they fill out this survey, they are taken to Screen 4 (second from right). They are assigned a random client ID and code which their parent can use to link their accounts. The parent is then given the opportunity to edit any of the health information inputted by the teen on Screen 5 (right-most).

\section{Conclusions}

The mood swing detection algorithm had a $96.0 \%$ accuracy which is higher than existing algorithms, indicating that the dual-model algorithm design may be more effective than a multiclass classification design. One limitation was lack of access to training data that consisted of messages sent during a prescription sti- 
mulant abuse episode. Access to data annotated with this information would improve algorithm performance. However, this problem was avoided to the extent possible by training the models using groups defined by guidelines adapted from DSM-5. This helped capture the extremity of emotion associated with prescription stimulant abuse rather than routine emotional fluctuations. The mood swing detection algorithm is applied to detect overdoses in real-time by monitoring a teen's outgoing SMS messages securely, scoring each one using both the euphoria and melancholy models, computing continuous score differences, and updating the total overdose score if an extreme mood swing is detected.

The biochemical test strip's diagnostic measure had a $90.62 \%$ accuracy, providing further evidence that an AuNP-based method is effective for real-time detection of amphetamine metabolite. The $\mathrm{pH}$-based control measure had an accuracy of $84.38 \%$, providing support for this technique as a method to ensure appropriate sweat volume. One limitation is the variability of sweat $\mathrm{pH}$ between individuals and the unpredictability of sweat excretion volume. Although slightly acidic sweat was accounted for and an average sweat excretion volume of $10 \mu \mathrm{l}$ was used for optimization, more comprehensive testing is required to ensure that the strip performs accurately across a wider range of conditions.

The vital sign measurement algorithm had an accuracy of $97.86 \%$ which is comparable to other PPG-based algorithms. Future testing with individuals across a wide range of ages and heights, with an emphasis on teens because they are the target demographic, is necessary to determine the efficacy of personalization and optimize the algorithm for the Hero system. Within the Hero mobile app, the vital sign algorithm takes a 5-second recording of a user's fingertip using the phone's back camera, processes the recording, and extrapolates vital signs using height, age, and gender information.

The mood swing detection algorithm, biochemical test strip, and vital sign measurement algorithm are integrated into a 5-factor system and Hero mobile app. One limiting factor was the inability to test under clinical conditions. While the simulated evaluations provide informative results, human testing is necessary to account for variable environments, unpredicted conditions, and human error. The total cost of implementing all factors of the Hero system would be less than 30 dollars, assuming that the user already has the host devices (phone and laptop mouse), making Hero an economically feasible solution. Additionally, the ease with which each factor can be integrated into a teen's daily life makes Hero a viable framework to detect stimulant abuse in real-time. The novelty of this study is in how emotional, biochemical, and physical factors are combined to comprehensively detect prescription amphetamine overdoses and prevent fatalities amidst the growing stimulant epidemic.

In the future, more twitter data could be gathered to create more robust Artificial Intelligence models for the mood swing detection algorithm. Additionally, samples of human sweat could be collected to evaluate the biochemical test strip and multiple color sensors could be integrated to create a field of detection, potentially resulting in more accurate color readings and faster detection. The 
Hero system could be modified for real-time detection of illicit drug or opioid misuse, and these modified frameworks may be useful in substance abuse treatment center.

\section{Acknowledgements}

I would like to acknowledge Mrs. Karol Higgins, Mrs. Amanda Martinez, and Dr. Varsha Sonawane. Their support with ordering materials and lab experimentation is greatly appreciated.

\section{Conflicts of Interest}

The author declares no conflicts of interest regarding the publication of this paper.

\section{References}

[1] National Institute on Drug Abuse (2020) Prescription Stimulants DrugFacts. http://www.drugabuse.gov/publications/drugfacts/prescription-stimulants

[2] National Institute on Drug Abuse (2020) What Is the Scope of Prescription Drug Misuse?

http://www.drugabuse.gov/publications/research-reports/misuse-prescription-drug s/what-scope-prescription-drug-misuse

[3] Prieur, N. (2017) National Adolescent Drug Trends in 2017: Findings Released. Institute for Social Research, University of Michigan. http://www.monitoringthefuture.org/data/data.html

[4] Weyandt, L.L., White, T.L., Gudmundsdottir, B.G., Nitenson, A.Z., Rathkey, E.S., De Leon, K.A. and Bjorn, S.A. (2018) Neurocognitive, Autonomic, and Mood Effects of Adderall: A Pilot Study of Healthy College Students. Pharmacy, 6, 58. http://www.ncbi.nlm.nih.gov/pmc/articles/PMC6165228/ https://doi.org/10.3390/pharmacy6030058

[5] Center for Disease Control (2018) 2018 Annual Surveillance Report of Drug-Related Risks and Outcomes. http://www.cdc.gov/drugoverdose/pubs/related-publications.html

[6] Arria, A.M. and DuPont, R.L. (2010) Nonmedical Prescription Stimulant Use among College Students: Why We Need to Do Something and What We Need to Do. Journal of Addictive Diseases, 29, 417-426.

http://www.ncbi.nlm.nih.gov/pmc/articles/PMC2951617/

https://doi.org/10.1080/10550887.2010.509273

[7] Meredith, G., DeLollis, M. and Shad, M.U. (2020) Potential Treatment for Overdose with Synthetic Cannabinoids. Medical Cannabis and Cannabinoids, Karger Publishers. http://www.karger.com/Article/FullText/506635

[8] National Institute on Drug Abuse (2020) Opioid Overdose Reversal with Naloxone (Narcan, Evzio).

http://www.drugabuse.gov/drug-topics/opioids/opioid-overdose-reversal-naloxonenarcan-evzio

[9] Facts about Overdose. Learn to Cope-A Support Organization That Offers Education, Resources, Peer Support and Hope for Parents and Family Members Coping with a Loved One Addicted to Heroin, Opioids or Other Drugs. http://www.learn2cope.org/facts-about-overdose/ 
[10] Nandakumar, R., Gollakota, S. and Sunshine, J.E. (2019) Opioid Overdose Detection Using Smartphones. Science Translational Medicine, 11, eaau8914. https://doi.org/10.1126/scitranslmed.aau8914

[11] Leyton, M., aan het Rot, M., Booij, L., Baker, G.B., Young, S.N. and Benkelfat, C. (2007) Mood-Elevating Effects of D-Amphetamine and Incentive Salience: The Effect of Acute Dopamine Precursor Depletion. Journal of Psychiatry \& Neuroscience, 32, 129-136.

[12] Baig, A.M. (2018) DARK Side of Amphetamine and Analogues: Pharmacology, Syndromic Manifestation, and Management of Amphetamine Addiction. ACS Chemical Neuroscience, 9, 2299-2303.

https://doi.org/10.1021/acschemneuro.8b00137

[13] National Institute on Drug Abuse (2020) Drugs and the Brain. https://www.drugabuse.gov/publications/drugs-brains-behavior-science-addiction/ drugs-brain

[14] Promises Behavioral Health. (2017). https://www.addiction.com/avoidance-drug-crash-motivate-de-motivate-cocaine-u $\underline{\text { se/ }}$

[15] Kalyani, Gupta, E., Rathee, G. and Chauhan, D.S. (2015) Mood Swing Analyser: A Dynamic Sentiment Detection Approach. Proceedings of the National Academy of Sciences, India Section A: Physical Sciences, 85, 149-157.

https://www.researchgate.net/publication/279288383_Mood_Swing_Analyser_A_D ynamic_Sentiment_Detection_Approach https://doi.org/10.1007/s40010-014-0169-x

[16] Hudson, M., Stuchinskaya, T., Ramma, S., Patel, J., Sievers, C., Goetz, S., Hines, S., Menzies, E. and Russell, D.A. (2019) Drug Screening Using the Sweat of a Fingerprint: Lateral Flow Detection of $\Delta 9$-Tetrahydrocannabinol, Cocaine, Opiates and Amphetamine. Journal of Analytical Toxicology, 43, 88-95.

https://doi.org/10.1093/jat/bky068

[17] Dring, L.G., Smith, R.L. and Williams, R.T. (1970) The Metabolic Fate of Amphetamine in Man and Other Species. The Biochemical Journal, 116, 425-435. https://doi.org/10.1042/bj1160425

[18] Barnes, A.J., Smith, M.L., Kacinko, S.L., Schwilke, E.W., Cone, E.J., Moolchan, E. T. and Huestis, M.A. (2008) Excretion of Methamphetamine and Amphetamine in Human Sweat Following Controlled Oral Methamphetamine Administration. Clinical Chemistry, 54, 172-180. https://doi.org/10.1373/clinchem.2007.092304

[19] Food and Drug Administration. (2007). https://www.accessdata.fda.gov/drugsatfda_docs/label/2007/011522s040lbl.pdf

[20] United States Office on Drugs and Crime (2014) Guidelines for Testing Drugs under International Control in Hair, Sweat, and Oral Fluid.

https://www.unodc.org/documents/scientific/ST_NAR_30_Rev.3_Hair_Sweat_and_ Oral_Fluid.pdf

[21] Yoo, Y.K., Kim, G., Park, D., et al. (2020) Gold Nanoparticles Assisted Sensitivity Improvement of Interdigitated Microelectrodes Biosensor for Amyloid- $\beta$ Detection in Plasma Sample. Sensors and Actuators B: Chemical, 308, 127710. https://doi.org/10.1016/j.snb.2020.127710

[22] Bariya, M., Li, L., Ghattamaneni, R., et al. (2020) Glove-Based Sensors for Multimodal Monitoring of Natural Sweat. Science Advances, 6, eabb8308. https://doi.org/10.1126/sciadv.abb8308

[23] Chen, Y.-L., Kuan, W.-H. and Liu, C.-L. (2020) Comparative Study of the Composi- 
tion of Sweat from Eccrine and Apocrine Sweat Glands during Exercise and in Heat. International Journal of Environmental Research and Public Health, 17, 3377.

http://www.ncbi.nlm.nih.gov/pmc/articles/PMC7277079/ https://doi.org/10.3390/ijerph17103377

[24] O’Donnell, J., et al. (2020) Vital Signs: Characteristics of Drug Overdose Deaths Involving Opioids and Stimulants-24 States and the District of Columbia, January-June 2019. MMWR Morbidity and Mortality Weekly Report, 69, 1189-1197. http://www.ncbi.nlm.nih.gov/pmc/articles/PMC7470457/

[25] Khanam, F.-T.-Z., Al-Naji, A. and Chahl, J. (2019) Remote Monitoring of Vital Signs in Diverse Non-Clinical and Clinical Scenarios Using Computer Vision Systems: A Review. Applied Sciences, 9, 4474.

http://www.mdpi.com/2076-3417/9/20/4474/htm https://doi.org/10.3390/app9204474

[26] Castaneda, D., Esparza, A., Ghamari, M., Soltanpur, C. and Nazeran, H. (2018) A Review on Wearable Photoplethysmography Sensors and Their Potential Future Applications in Health Care. International Journal of Biosensors \& Bioelectronics, 4, 195-202. http://www.ncbi.nlm.nih.gov/pmc/articles/PMC6426305/

[27] Lazazzera, R., Belhaj, Y. and Carrault, G. (2019) A New Wearable Device for Blood Pressure Estimation Using Photoplethysmogram. Sensors (BaseI), 19, 2557. http://www.ncbi.nlm.nih.gov/pmc/articles/PMC6603632/

[28] Dreaden, E.C., Alkilany, A.M., Huang, X.H., Murphy, C.J. and El-Sayed, M.A. (2012) The Golden Age: Gold Nanoparticles for Biomedicine. Chemical Society Reviews, 41, 2740-2779. http://www.ncbi.nlm.nih.gov/pmc/articles/PMC5876014/

[29] Castro, W., Oblitas, J., Santa-Cruz, R. and Avila-George, H. (2017) Multilayer Perceptron Architecture Optimization Using Parallel Computing Techniques. PloS One, 12, e0189369. https://doi.org/10.1371/journal.pone.0189369

[30] Gaind, B., Syal, V. and Padgalwar, S. (2019) Emotion Detection and Analysis on Social Media. Global Journal of Engineering Science and Research, 78-89. https://arxiv.org/pdf/1901.08458.pdf 